\title{
Terahertz radiation in two-color laser fields: from single atom to extended gas response
}

\author{
S. Yu. Stremoukhov ${ }^{1,2}$, A.V. Andreev ${ }^{1}$ \\ ${ }^{1}$ Faculty of Physics, Lomonosov Moscow State University, Leninskie Gory, 1, build.2, 119991, Moscow, Russia, \\ sustrem@gmail.com \\ ${ }^{2}$ National Research Centre “Kurchatov Institute”, pl. Akademika Kurchatova, 1, 123182, Moscow, Russia
}

One of the promising methods of the $\mathrm{THz}$ radiation generation is the use of the two-color laser field focused into the gas media $[1,2]$. The polarization of the low frequency $\mathrm{THz}$ field generated in gas is linear [2], although, in some conditions, it is elliptical due to the modulation of the laser phase and its polarization in the gas plasma [3]. At the same time, for some practical applications, it is critical to have the elliptically polarized $\mathrm{THz}$ radiation [4]. For its generation certain methods $[2,5]$ mostly based on the mixing of the elliptically polarized laser fundamental radiation $(\omega)$ with linear (or elliptically polarized) second harmonic radiation $(2 \omega)$ exist, the generated elliptical $\mathrm{THz}$ radiation lies in low frequency region $(<5 \mathrm{THz})$.

Here, we demonstrate the method of generation of the highly elliptical high-frequency $\mathrm{THz}$ radiation ( $>25 \mathrm{THz}$ ) by the extended gas media interacting with the two-color laser field having linearly polarized components but with a nonzero angle between them [6]. The consistent quantum-mechanical description is given. The two spatial levels are introduced. Initially, the time-dependent Schrodinger equation for single atom "placed" in a finite number of positions in the extended gas (i.e. being under the action of the twocolor laser field having various intensities and relative phases between the pulses [7]) is solved. Then, we calculate the response of extended system by changing the spatial parameters of the gas (mainly, the gas length, $l$ ) and the laser beam (mainly, beam width, $\sigma$ )).

For the calculation of the properties of the $\mathrm{THz}$ radiation emitted by a single atom we have used the non-perturbative theory $[8,9]$ and the model of the $\mathrm{Ar}$ atom described in $[7,10]$. In simulations we assume that the components of the two-color laser field have the fixed intensity $\left(I_{\omega}=6.77 \times 10^{12} \mathrm{~W} \cdot \mathrm{cm}^{-2}\right.$ and $I_{2 \omega}=$ $\left.5.85 \times 10^{11} \mathrm{~W} \cdot \mathrm{cm}^{-2}\right)$, fixed temporal width of the laser field components $\left(\tau_{1,2}=30 \mathrm{fs}\right)$, zero delay time between the pulses $\left(\Delta t_{1,2}=0\right)$ and relative phase equal to $\varphi_{0}=\pi / 2$. At these parameters of laser field the ionization-free regime of interaction occurs [10]. In this way we obtain a series of photoemission spectra for different angles between the polarizations of two-color laser field components $(\theta)$ and different delay times between the pulses.

Single atom numerical calculations demonstrate that at frequences $>25 \mathrm{THz}$ there are some regions of high ellipticity (up to circular), the maximum value of the ellipticity and the widths of these regions decrease along with an increase in the $\mathrm{THz}$ frequency [6].

Basing on the single-atom response we have calculated the response of the extended gas media. Previously we have used the formula (4) from [7] for describing the linearly polarized $\mathrm{THz}$ laser field spa- tial distribution in an aria around the gas. In that calculations we have summarized two projections onto perpendicular axes of $\mathrm{THz}$ fields emitted from individual atoms and have extracted total intensity of the $\mathrm{THz}$ radiation at given points of the area. In current calculations of the elliptically polarized $\mathrm{THz}$ field response each atom emits elliptically polarized radiation which could be expanded on two projections on the perpendicular axes $E_{x j}=\left|E_{x j}\right| e^{i \sigma_{x j}} \quad$ and $E_{y j}=\left|E_{y j}\right| e^{i \sigma_{y j}}$ (see Fig. 1).
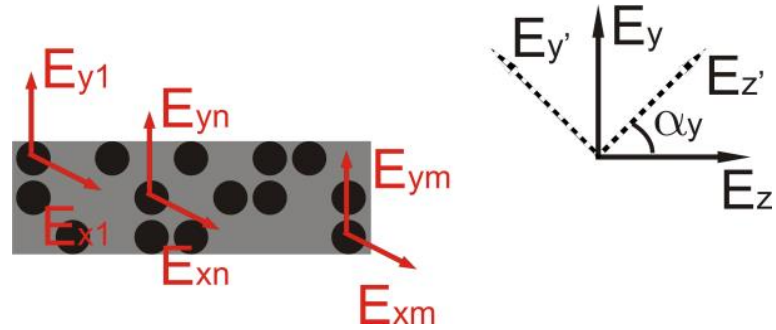

Fig. 1 Schematic view of the gas (gray rectangle) with atoms (black circles) emitting the THz field (red arrows indicate two projections). By the black solid arrows and black dashed arrows indicated the two projections of the field formed by the sum of projections of the single color field on the one axes. $\alpha_{y}$ denotes the rotation angle.

By using formulae (4) from [7] two projections of the summarized field could be found at given point of the area for each projections $(x-$ and $y-)$ of the $\mathrm{THz}$ field separately (see Fig.1 with shown projections only for $y$ - component of the $\mathrm{THz}$ field, the other two projections for $x$ - component of the $\mathrm{THz}$ field lies in the perpendicular plane). As a result, two projections of the single atom $\mathrm{THz}$ field response are transformed at four ones when the problem of extended gas media response is under consideration. But the turning of two perpendicular axes on the angle can decrease the number of projections. Indeed, if we turn $y z-$ axes on the angle $\alpha$, the projections of the $\mathrm{THz}$ field on the new $y^{\prime} z^{\prime}-$ axes become

$$
\begin{aligned}
& E_{y^{\prime}}=E_{y} \cos \alpha+E_{z} \sin \alpha, \\
& E_{z^{\prime}}=-E_{y} \sin \alpha+E_{z} \cos \alpha .
\end{aligned}
$$

If the rotation angle will be chosen by the following way

$$
\tan \alpha_{y}=\frac{E_{z}}{E_{y}},
$$

then $E_{z^{\prime}}=0$ and $E_{y^{\prime}}$ will have the form of

$$
E_{y^{\prime}}=E_{y} \cos \left(\arctan \left(\frac{E_{z}}{E_{y}}\right)\right)+E_{z} \sin \left(\arctan \left(\frac{E_{z}}{E_{y}}\right)\right) \text {. }
$$


As a result, only one complex projection have nonzero value. Providing the following procedure with other two projections (with are corresponding to the $x$-component of the atoms $\mathrm{THz}$ components) we will have only one projection of the $\mathrm{THz}$ field which has the form of

$$
E_{x^{\prime}}=E_{x} \cos \left(\arctan \left(\frac{E_{z}}{E_{x}}\right)\right)+E_{z} \sin \left(\arctan \left(\frac{E_{z}}{E_{x}}\right)\right) \text {. }
$$

So, there are only two complex projections of the $\mathrm{THz}$ field at each point of space. Using them and providing some standard procedures ones can extract properties of the $\mathrm{THz}$ field (intensity, ellipticity, polarization angle).
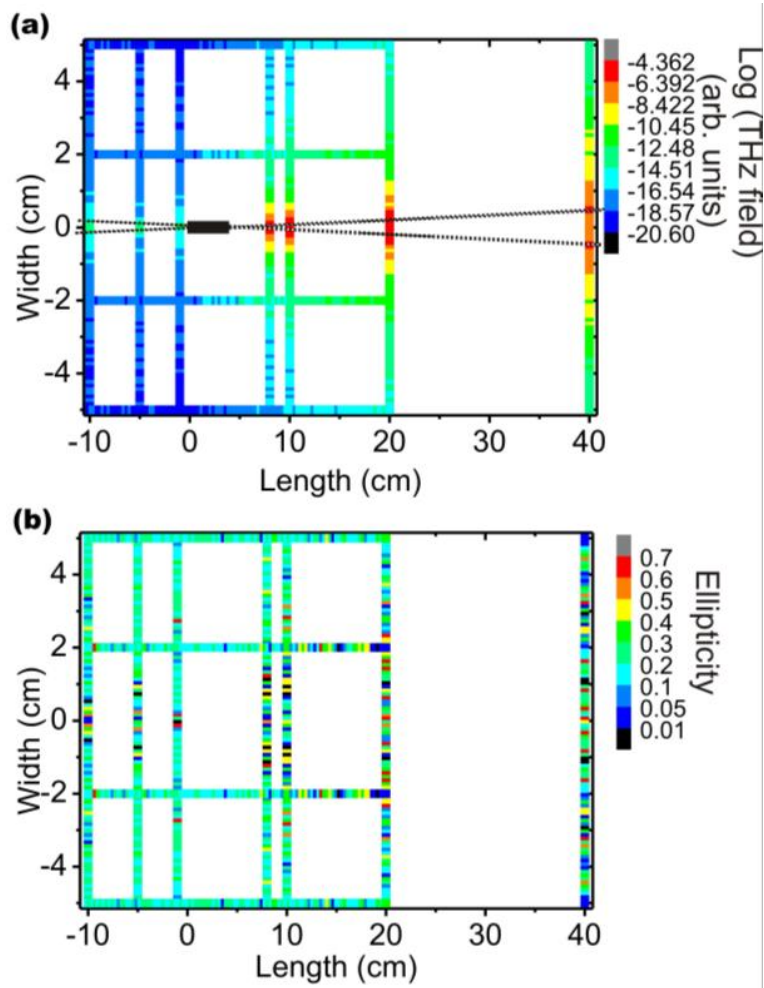

Fig. 2 Spatial distribution of the $\mathrm{THz}$ field strengths (a) and ellipticities (b) calculated for $v=29 \mathrm{THz}$ for $1=6 \mathrm{~cm}$ and $\sigma=0.1 \mathrm{~cm}$. The black rectangle is the emitted medium.

The typical $\mathrm{THz}$ field distributions calculated for $l=6 \mathrm{~cm}, \sigma=0.1 \mathrm{~cm}, \theta=\pi / 4$ and $v=29 \mathrm{THz}$ are presented in Fig. 2. The THz field distributions demonstrate the cone structure mainly. These distributions also contain the conical structure in the backward direction [11], which is possible due to the complete orders in "emitted atoms" locations in the gas (with a fixed period). The backward emission is less intensive compared to the forward one. Fig. 2b demonstrated the ellipticity distribution of the generated radiation.

We have investigated three possibilities to control the value of ellipticity (and the $\mathrm{THz}$ field strength) of the generated radiation. The first one is to change the relative phase between the pulses of the two-color laser field at the left side of the gas (the same method was previously investigated in [2]).

The second way to an effective control of the value of the ellipticity of the generated $\mathrm{THz}$ radiation is to change the spatial geometry of the interaction (the length of the gas $l$ and the width of the laser beam $\sigma$ ).

The third method of the effective control of the ellipticity value of the generated $\mathrm{THz}$ radiation is by changing the spatial profile of the laser beam, i.e. by using an iris and a mask which is completely opposite to the iris: the iris transmits, in this case, the central part of the laser pulses, and the mask transmits the periphery one [6].

The work was partially supported by the Russian Foundation for Basic Research under Projects Nos. 18-02-00528, 18-02-00743, 18-02-40014.

\section{References}

1. Cook, D. J., Hochstrasser, R. M. Intense terahertz pulses by four-wave rectification in air // Opt. Lett. 2000. V. 25 , p. 1210

2. Dai, J., Karpowicz, N., Zhang ,X. C. Coherent Polarization Control of Terahertz Waves Generated from TwoColor Laser-Induced Gas Plasma // Phys. Rev. Lett. 2009. V. 103, p. 023001.

3. Xu, J., Galan, J., Ramian, G., Savvidis, P., Scopatz, A., Birge, R. R., Allen, S. J., Plaxco, K. Terahertz Circular Dichroism Spectroscopy of Biomolecules // Proc. SPIE. 2004. 5268, 19-26.

4. Hughes, S., Citrin, D.S. Interaction of terahertz transients and broadband optical pulses in quantum wells // J. Opt. Soc. Am. B. 2000. V. 17, p. 128.

5. Sato, M., Higuchi, T., Kanda, N., Konishi, K., Yoshioka, K., Suzuki, T., Misawa, K., Kuwata-Gonokami, M. Terahertz polarization pulse shaping with arbitrary field control // Nat. Photonics. 2013. V. 7, p. 724.

6. Stremoukhov, S., Andreev, A. Quantum-mechanical fingerprints in generation of elliptical terahertz radiation by extended media interacting with two-color laser field // J. Opt. Soc. Am. B. 2017. V. 34(2), p. 232-237.

7. Stremoukhov, S. Yu., Andreev, A. V. Spatial variations of the intensity of $\mathrm{THz}$ radiation emitted by extended media in two-color laser fields // Laser Phys. Lett. 2015. V. 12, p. 015402.

8. Andreev, A.V., Stremoukhov, S. Yu., Shoutova, O. A. Light-induced anisotropy of atomic response: prospects for emission spectrum control // Eur. Phys. J. D. 2012. V. 66, 16.

9. Stremoukhov, S., Andreev, A., Vodungbo, B., Salières, $P$., Mahieu, B., Lambert, G. Origin of ellipticity of high-order harmonics generated by a two-color laser field in the cross-polarized configuration // Phys. Rev. A. 2016. V. 94, p. 013855.

10. Andreev, A. V., Stremoukhov, S. Yu. Terahertzradiation generation in the ionization-free regime of lightatom interaction // Phys. Rev. A. 2013. V. 87, p. 053416.

11. Jahangiri, F., Hashida, M., Nagashima, T., Tokita, S., Hangyo, M., Sakabe, S. Intense terahertz emission from atomic cluster plasma produced by intense femtosecond laser pulses // Appl. Phys. Lett. 2011. V. 99, p. 261503. 